\title{
Galaxy cluster formation from the large-scale structure: a case study of the Abell 2125 complex at $\mathrm{z}=0.247$
}

\author{
Q. Daniel Wang ${ }^{1}$, Frazer Owen ${ }^{2}$, Michael Ledlow ${ }^{3}$ and William Keel ${ }^{4}$ \\ ${ }^{1}$ Department of Astronomy, University of Massachusetts, Amherst, MA, USA \\ ${ }^{2}$ National Radio Astronomy Observatory, Scorro, NM, USA \\ ${ }^{3}$ Gemini Observatory, La Serena, Chile \\ ${ }^{4}$ Department of Astronomy, University of Alabama, Tuscaloosa, AL, USA
}

\begin{abstract}
The structure of the universe is believed to have formed by clustering hierarchically from small to large scales. Much of this evolution occurs very slowly but at a few special times more rapid, violent activity may occur as major subunits collide at high velocities. The Abell 2125 complex $(z=0.247)$ appears to be undergoing such an event as shown by modeling of the optical velocity field and by the detection with the VLA of an unusually large number of associated radio active galaxies. We present an $80 \mathrm{ksec}$ Chandra imaging of Abell 2125, together with extensive complementary multi-wavelength data. We show direct evidence for galaxy transformation and destruction during the cluster formation. The Chandra data unambiguously separate the X-ray contributions from discrete sources and large-scale diffuse gas in the Abell 2125 complex, which consists of various merging clusters/groups of galaxies and low-surface brightness emission. This enables us to study processes affecting galaxy evolution during this special time from scales of $\mathrm{Mpc}$ down to a few kpc. The overall level of activity plus the special time for the cluster-cluster merger suggests that an important phase of galaxy evolution can take place during such events.
\end{abstract}

\section{Introduction}

We have identified a large-scale hierarchical complex (Fig. 1 left panel) that is wellsuited for investigating the structure formation process and the galaxy/environment interactions. Revealed in a survey of 10 Butcher \& Oemler clusters observed with the ROSAT PSPC, this complex contains various X-ray-emitting features, which are associated with concentrations of optical and radio galaxies (Fig. 1; Wang, Connolly, \& Brunner 1997; Wang, Owen, \& Ledlow 2004). The overall optical galaxy concentration of the region was classified previously as a cluster Abell 2125 (richness 4). In addition to its large blue galaxy fraction $(\sim 20 \%)$, Abell 2125 also contains an unusually high number of radio sources, a factor of a few higher than those in more-or-less relaxed clusters at about the same redshifts. The ROSAT image and follow-up optical observations have further shown that the complex contains three well-defined X-ray bright clusters (Wang et al. 1997). In addition, substantial amounts of unresolved low-surface brightness X-ray emission (LSBXE) are also present. The overall size of the complex is at least $\sim 5 \mathrm{Mpc}$ (assuming the now standard $\Lambda \mathrm{CDM}$ cosmology). The complex thus represents an X-raybright hierarchical filamentary superstructure, as predicted by numerical simulations of the structure formation (e.g., Cen \& Ostriker 1996).

We have obtained an $80 \mathrm{ksec}$ Chandra observation that covers part of the Abell 2125 complex (Fig. 1, right panel) to characterize its detailed X-ray properties and to study the interplay between galaxies with their environments. The high spatial resolution of 

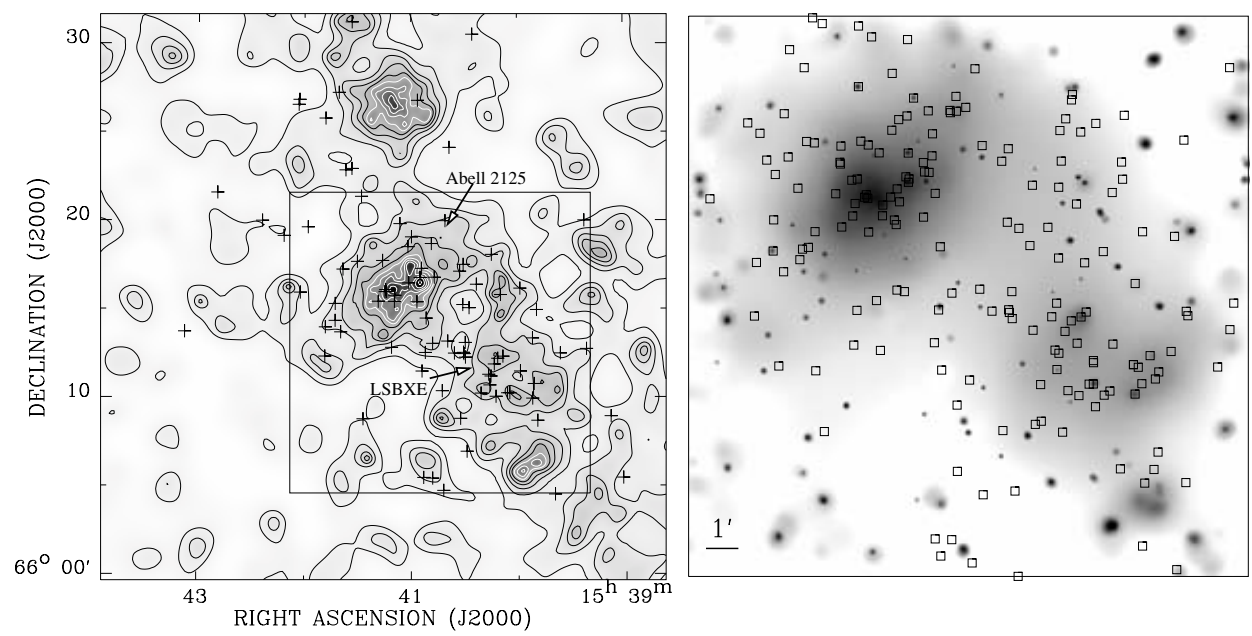

Figure 1. ROSAT PSPC X-ray image of the Abell 2125 complex in the $0.5-2 \mathrm{keV}$ band (left panel; Wang et al. 1997). Point-like X-ray sources detected in the image have been excised. Spectroscopically confirmed, radio detected members of the Abell 2125 complex (Owen et al. 2004a) are marked by pluses. The box outlines the field covered by our Chandra ACIS-I observation shown in the right panel, where the intensity image is in the $0.5-2 \mathrm{keV}$ band. Squares mark the positions of complex member galaxies.

Chandra enables us to detect point-like sources down to a limit of $\sim 1 \times 10^{-15} \mathrm{erg} \mathrm{cm}^{-2}$ $\mathrm{s}^{-1}$ in the $0.5-8 \mathrm{keV}$ band and to examine diffuse $\mathrm{X}$-ray structures down to a scale of $\sim 3.8 \mathrm{kpc}\left(\sim 1^{\prime \prime}\right)$. Here we summarize results from the analysis of the data and from the comparison with extensive multi-wavelength observations of the complex.

\section{New results}

We detect a total of 99 discrete sources in the field. Ten of these sources are identified with optical members of the complex. A statistical analysis shows that few (if any) of the remaining unidentified sources are likely to be members of the complex. The diffuse emission is substantially softer than typical discrete sources. There are substructures in the diffuse X-ray emission on all scales. The large-scale diffuse X-ray emission is correlated well with identified complex member galaxies (e.g., Fig. 1 right panel). Two relatively prominent concentrations are sampled by the ACIS-I observation: the Abell 2125 cluster and the southwest LSBXE patch. The redshifts obtained from our X-ray spectra of these features are consistent with the optical value $(z=0.247)$ of the complex.

\subsection{Nature of the $L S B X E$}

The LSBXE is distinctly different from the central cluster. Even the relatively X-raybright southwest patch does not seem to be centrally peaked, as would be expected from a more-or-less virialized intracluster medium (ICM). The kinematics (Miller et al. 2004) indicates that the galaxies in this patch are probably loosely bound, although their exact line-of-sight distribution is not clear. Fig. 2 shows a sub-field of the LSBXE. There are three bright ellipticals in the field; but most of the other galaxies appear to be spirals. X-ray emission from such galaxies are typically dominated by X-ray binaries and/or AGNs and is therefore expected to have a relatively hard spectrum. But the diffuse 

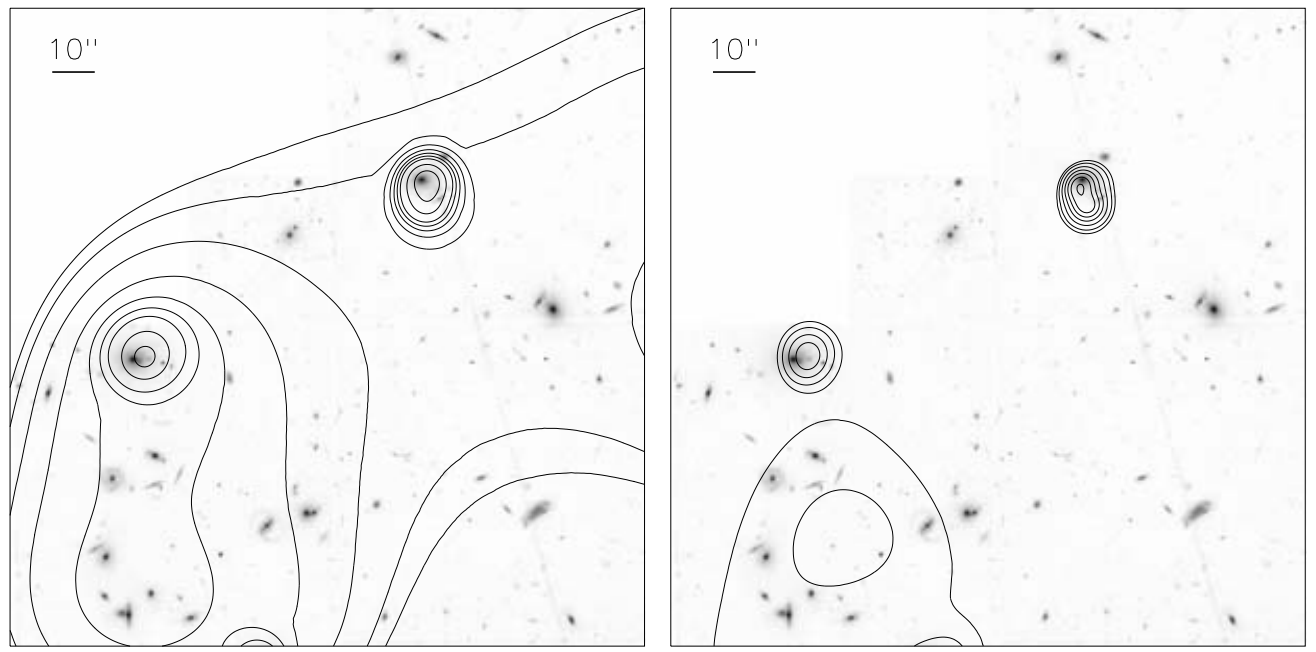

Figure 2. HST WFPC-2 V-band images of a field in the LSBXE and overlaid X-ray contours in the the $0.5-2 \mathrm{keV}$ band (left panel) and the $2-8 \mathrm{keV}$ band (right panel).
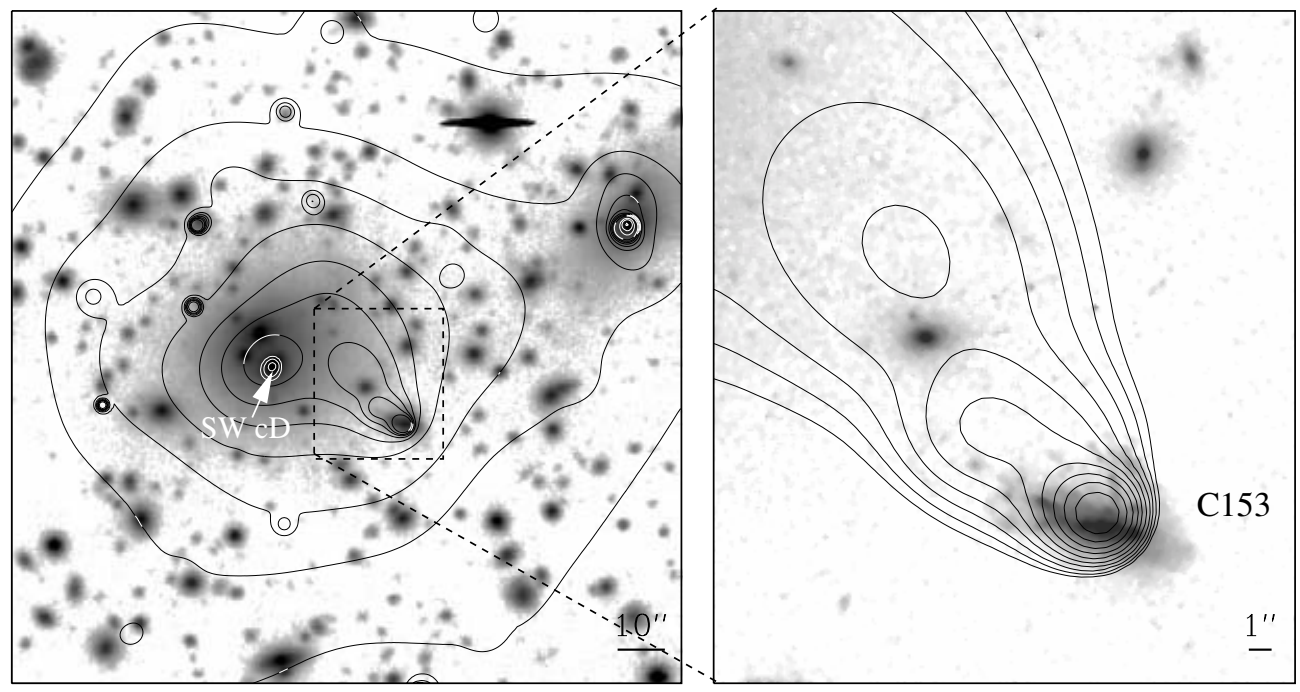

Figure 3. ACIS-I intensity images of the central cluster in the Kitt Peak 4-m mosaic V-band (red) as well as in the $0.5-2 \mathrm{keV}$ (green) and $2-8 \mathrm{keV}$ (blue) bands (left panel); (right panel) a close-up of C153 in an HST WFPC-2 V-band (red) and its diffuse X-ray trail.

X-ray enhancement associated with the galaxy concentration is soft, indicating an origin in diffuse hot gas around individual galaxies, in groups of galaxies, and/or in the intergroup IGM.

The heavy element abundance in the LSBXE $(<10 \%$ solar $)$ appears to be substantially lower than that in the ICM of the central cluster $(\sim 1 / 4$ solar $)$, although the abundance may be underestimated in the LSBXE if it contains multiple temperature components. If the low metallicity obtained from the single temperature plasma fit is real, there may be two possible explanations: 1) Metals (mainly irons) are largely locked in dust grains within or around galaxy groups; 2) The metals are still bound in individual galaxies. Dust grains may survive, and may even not mix well with, the X-ray-emitting gas in a 
quiescent, low density environment. But the mixing should likely occur during or after the merger of a galaxy or group with a cluster. The dust grains would then be destroyed rapidly by sputtering in the ICM of high density and temperature. This may explain the metal abundance difference in the X-ray-emitting gas between the LSBXE and the central cluster.

\subsection{Dynamical state of the central cluster}

The central cluster (Abell 2125; Fig. 1) represents the strongest enhancement of the diffuse X-ray emission in the complex. The relative values of our measured temperature $(3 \mathrm{keV})$ and luminosity of the ICM $\left(\sim 7.9 \times 10^{43} \mathrm{ergs} \mathrm{s}^{-1}\right.$ in the $0.5-2 \mathrm{keV}$ band $)$ are consistent with those for typical clusters. But, the presence of strong substructures in both the X-ray morphology and galaxy kinematics indicates that the cluster is undergoing a merger.

The cluster also represents the densest concentration of galaxies in the Abell 2125 complex. Although most of the associated galaxies are quiet in radio, the brightest radio galaxies in the field are found in the core of the cluster. While the galaxy C153 is a disturbed disk-like galaxy, the other three are all cD-like ellipticals (Wang et al. 2004; Owen et al. 2004b). Interestingly, the two galaxies (C153 and SW cD; Fig. 3) associated with enhanced X-ray emission are both moving at high velocities $\left(\sim 1.5-1.8 \times 10^{3} \mathrm{~km} \mathrm{~s}^{-1}\right)$ relative to the mean of the cluster.

The exact relationship between the LSBXE and the central cluster is not clear. The similar velocity centroids of the associated galaxy concentrations may indicate that they are about to merge along an axis that is perpendicular to the line of sight, although there is little direct evidence from both X-ray and galaxy kinematics.

\subsection{Galaxy-environment interaction}

Most of the $10 \mathrm{X}$-ray-bright member galaxies are not resolved by Chandra and are probably dominated by AGN activities. Two of the X-ray sources are apparently resolved and are associated with the disturbed disk-like galaxy C153 and the giant elliptical galaxy northwest of the central Abell 2125 cluster (Fig. 3 left panel). As our source detection algorithms are optimized to detect point-like sources, extended sources such as the one associated with the SW cD (Fig. 3 left panel) are not detected. These extended soft X-ray enhancements probably represent hot gas associated with individual massive galaxies or groups of galaxies, which may have entered the cluster for the first time. Outside the central cluster, the ambient density and relative velocity are typically low and the ram-pressure stripping is probably not important, at least for massive galaxies. The intergalactic gas around galaxies may even cool fast enough to replenish the gas consumed for star formation (Bekki et al. 2002). As they are plugging into a cluster, the surrounding gas may then be compressed by the high ram-pressure of the ICM, resulting in enhanced soft X-ray emission. The eventual stripping of the gas and dust may be important in both enriching the ICM and transforming the galaxies.

The most conspicuous soft X-ray feature in the core of the central cluster is a "trail" apparently attached to the radio galaxy C153 (Fig. 3 right panel). A similar, though shorter, trail in the same direction is also seen in [OII] line emission (Owen et al. 2004b). C153 probably represents an extreme case of the ram-pressure stripping. The observed steep X-ray spectral characteristics, as evidenced by the complete absence of the trail in the $2-8 \mathrm{keV}$ band, suggests a thermal gas temperature of $\sim 0.7 \mathrm{keV}$, although the counting statistics of the data is not sufficient to give a tight constraint. We estimate the total luminosity of the trail as $\sim 5 \times 10^{41} \mathrm{ergs} \mathrm{s}^{-1}$ in the $0.5-2 \mathrm{keV}$ band. 


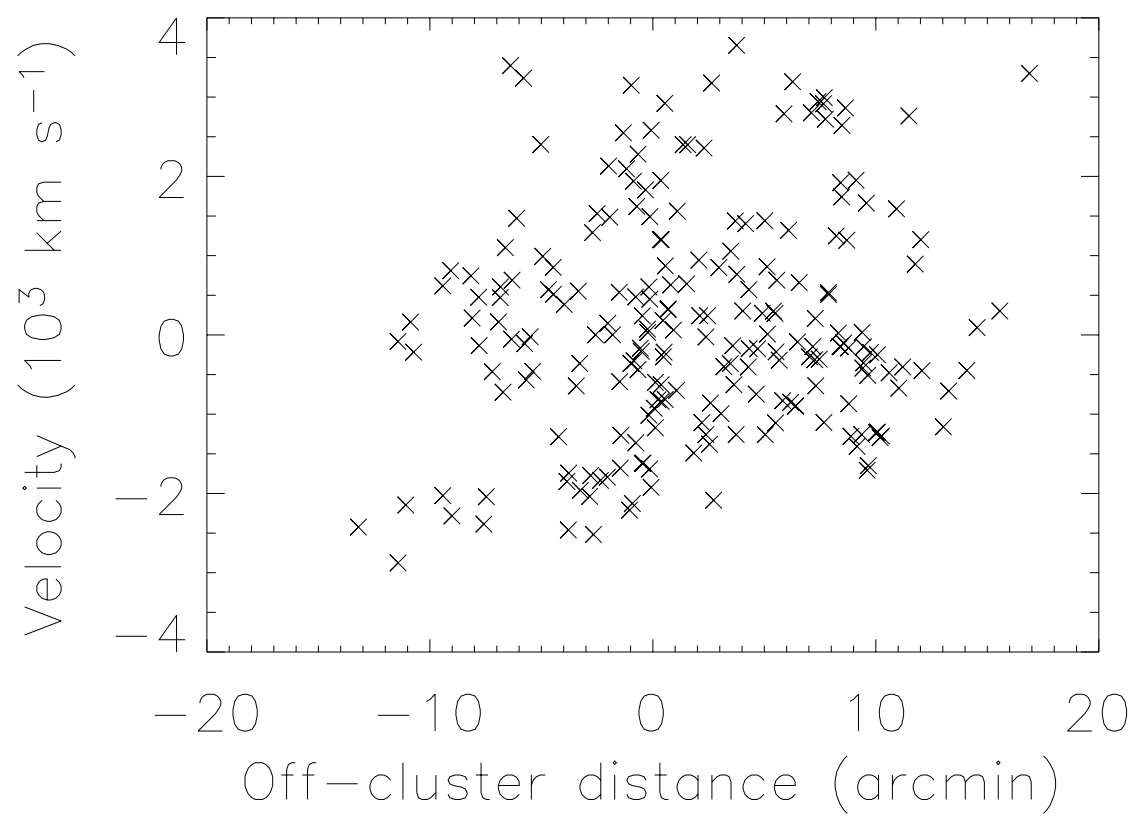

Figure 4. Radial velocities of galaxies versus the distance from the core cluster along the major axis $\left(\sim 40^{\circ}\right.$ east from the north) of the Abell 2125 complex.

\subsection{Abell 2125 as a large-scale hierarchical complex}

This distinct complex of galaxies and hot gas shows several remarkable characteristics:

- an unusually large galaxy velocity dispersion of $\sim 1.1 \times 10^{3} \mathrm{~km} \mathrm{~s}^{-1}$, with multiple velocity components over a scale of $\sim 5 \mathrm{Mpc}$ (e.g., Fig. 4);

- an exceptionally large fraction of radio galaxies, which are located primarily outside the central Abell 2125 cluster (Owen et al. 2004a);

- the presence of multiple X-ray-emitting clusters and LSBXE features, each with substantially lower luminosity and temperature than expected from the overall galaxy richness and large velocity dispersion of the complex (Wang et al. 2004; Wang et al. 1997).

Comparisons with various numerical simulations indicate that the complex represents a projection of multiple components, which might be in a process of merging with each other (Miller et al. 2004; Owen et al. 2004a). Projection effects, together with enhanced activities during this process, may explain the observed characteristics.

\section{References}

Bekki, K., Couch, W. J., \& Shioya, Y. 2002, ApJ, 577, 651

Cen, R., \& Ostriker, J. P. 1999, ApJ, 514, 1

Miller et al. 2004, ApJ, submitted

Owen, F. N., et al. 2004a, in preparation

Owen, F. N., et al. 2004b, in preparation

Wang, Q. D., Connolly, A., \& Brunner, R. 1997, ApJL, 487, 13

Wang, Q. D., et al. 2004, ApJ, submitted 